
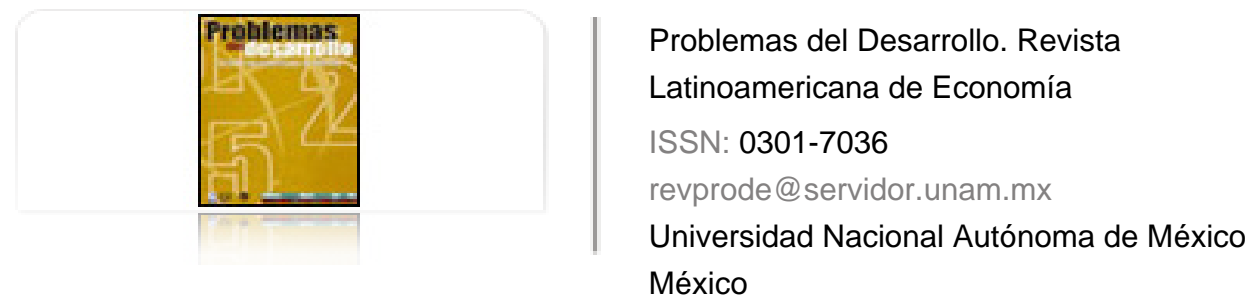

BRICEÑO RUIZ, JOSÉ

El modelo TLCAN de integración y las negociaciones del ALCA

Problemas del Desarrollo. Revista Latinoamericana de Economía, vol. 32, núm. 126, abril-julio, 2001, pp. $35-57$

Universidad Nacional Autónoma de México

Distrito Federal, México

Disponible en: http://www.redalyc.org/articulo.oa?id=11820094003

- Cómo citar el artículo

- Número completo

- Más información del artículo

Página de la revista en redalyc.org

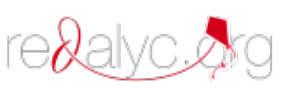

Sistema de Información Científica

Red de Revistas Científicas de América Latina, el Caribe, España y Portugal Proyecto académico sin fines de lucro, desarrollado bajo la iniciativa de acceso abierto 


\section{EL MODELO TLCAN DE INTEGRACIÓN}

\section{Y LAS NEGOCIACIONES DEL ALCA}

JOSÉ BRICEÑO RUIZ *

\section{RESUMEN}

En este ensayo se analiza el Tratado de Libre Comercio de América del Norte (TLCAN) como modelo de integración para el proceso negociador del Área de Libre Comercio de las Américas (ALCA). Mediante un examen de la evolución de la propuesta de integración hemisférica, se considera el proyecto estadounidense de ampliar el modelo de integración que inspiró la creación del TLCAN, al resto de América Latina y el Caribe. De igual manera, se analiza la reacción de los países del Mercado Común del Sur (Mercosur), que bajo el liderazgo de Brasil han rechazado la pretensión de Estados Unidos de imponer el modelo TLCAN. En una primera parte del artículo, se estudian los diferentes modelos comerciales e intervencionistas de integración regional, en particular la Unión Europea y el regionalismo abierto de la Comisión Económica para América Latina (CEPAL). En la segunda parte, se evalúa el TLCAN como un nuevo modelo que tiene aspiraciones más ambiciosas que los tradicionales modelos comerciales. Se considera luego el debate sobre los modelos en el contexto de las negociaciones del ALCA y la respuesta del Mercosur. Finalmente, se concluye con algunas reflexiones sobre el destino del ALCA y la necesidad de que Estados Unidos reconozca la existencia de propuestas distintas al modelo TLCAN, requisito para una satisfactoria conclusión del proceso negociador.

Palabras clave: integración, ALCA, TLCAN, Mercosur.

* Candidato a doctor en ciencia política e investigador del Centre de Recherches sur 1' Amérique Latine (CREALC), IEP Aix-en Provence, Francia, <bricenoruis@yahoo.fr>. 


\section{THE NAFTA MODEL OF INTEGRATION \\ AND THE FTAA NEGOTIATIONS \\ ABSTRACT}

This paper analyzes the North American Free Trade Agreement (NAFTA) as a model of regional integration, together with its influence on the negotiating process of the Free Trade Area of the Americas (FTAA). The various commercial and interventionist models of integration, particularly the European Economic Commission for Latin America's open regionalism are evaluated in the first section of the paper. The nature of NAFTA as a new model of integration is analyzed in the second section, while the debate concerning the model to be implemented in the FTAA and the extent to which Mercosur has rejected the NAFTA model is considered in the third section. The paper ends with a series of conclusions.

Key words: integration, FTAA, NAFTA, Mercosur.

\section{LE MODÈLE D'INTÉGRATION DE L'ALENA ET LES NÉGOCIATIONS PORTANT SUR LA ZLEA RÉSUMÉ}

L'article analyse l'Accord de libre-échange nord-américain (ALENA) comme modèle d'intégration pour le processus de négociation de la Zone de libreéchange des Amériques (ZLEA). Le projet américain d'élargissement au reste de l'Amérique latine et des Caraibes du modèle d'intégration qui sous-tend l'ALENA est examiné à la lumière de l'évolution de la proposition d'intégration à l'échelon continental. S'y trouve également analysée la réaction des pays du Marché commun du Sud (Mercosur) qui, sous la houlette du Brésil, ont rejeté les prétentions des États-Unis à imposer le modèle ALENA. Dans une première partie sont étudiés les différents modèles d'intégration régionale aux niveaux échanges et intervention, notament l'Union européenne, et le régionalisme ouvert de la Commission économique pour l'Amérique latine et les Caraibes (CEPALC). La deuxième partie analyse l'ALENA en tant que nouveau modèle, aux aspirations plus ambitieuses que les modèles d'échange traditionnels. Est ensuite examiné le débat sur les modèles dans le contexte des négociations de la ZLEA et la réponse du Mercosur. Y figurent enfin quelques réflexions sur la destinée de la ZLEA et sur le fait que les États-Unis doivent reconnaître qu'il existe des propositions distinctes du modèle ALENA, condition indispensable pour une conclusion satisfaisante du processus de négociation.

Mots-clés: intégration, ZLEA, ALENA, Mercosur. 

grado de interdependencia entre Estados Unidos y México estaba produciendo una integración silenciosa, que en parte operaba fuera de la ley, "y destinada a incrementarse en los próximos veinte años". Diez años más tarde, en noviembre de 1993, luego de un extenso y polémico debate, el Congreso estadounidense ratificaba el Tratado de Libre Comercio de América del Norte (TLCAN), con lo cual concluía la etapa de integración silenciosa y se iniciaba el proceso de integración formal de la región norteamericana. En este ensayo se analiza el TLCAN como un nuevo modelo de integración, se examinan sus características principales y sus diferencias con los modelos tradicionales, en particular el modelo europeo y el regionalismo abierto de la Comisión Económica para América Latina (CEPAL). Finalmente, se estudia la validez del modelo TLCAN para el resto del hemisferio, en el contexto de las actuales negociaciones para establecer un Área de Libre Comercio de las Américas (ALCA).

\section{EL TLCAN Y LOS MODELOS DE INTEGRACIÓN}

El debate económico tradicional sobre los modelos de integración ha dividido a la doctrina entre los defensores de un enfoque comercial neoliberal y los que apoyan un modelo dirigista o intervencionista. Ciertamente, han existido en la práctica modelos eclécticos que combinan políticas intervencionistas y comerciales, variando el carácter de la mixtura según el proceso de integración. Existe además el modelo de planificación centralizada, pero éste carece de referente empírico después del colapso del mundo comunista.

El modelo comercialista suscribe las ideas de la teoría tradicional del comercio internacional, considera la integración como un segundo óptimo, como "una experiencia local de desvalorización de las fronteras, justificable en tanto que etapa transitoria que serviría para ir eliminando, escalonadamente, los aranceles y otras restricciones a los intercambios comerciales". ${ }^{2}$ En tal sentido, la integración debería dirigirse esencialmente a abolir las barreras y los obstáculos que impiden el libre flujo de bienes y servicios entre las diversas economías nacionales. De esta manera, se crearía un espacio económico integrado en el cual la actuación de las leyes del mercado permitiría intensificar la interdepen-

1 Clark Reynolds citado en Alejandro Chanona Burguette (1993), "Una reconsideración de las teorías de la integración y el problema de la integración económica formal entre México y Estados Unidos", Relaciones Internacionales, vol. xv, núm. 60, nueva época, México, octubre-diciembre, 1993, p. 91.

2 Juan Mario Vacchino, "Teorías, esquemas y experiencias de integración económica regional”, Mundo Nuevo, año 5, núm. 15-16, Caracas, enero-junio de 1982, pp. 154-182. 
dencia económica entre los países socios. En la literatura comercialista la integración debe realizarse por etapas, la primera de las cuales sería la zona de libre comercio hasta alcanzarse una unión económica total, previa consecución de una unión aduanera y un mercado común.

En los enfoques comercialistas la integración no ha sido siempre sinónimo de bienestar. Con base en los prístinos trabajos de Jacob Viner ${ }^{3}$ sobre las uniones aduaneras, se distingue entre los procesos de integración que favorecen la creación de comercio y aquellos que promueven su desviación. La creación de comercio supone el desplazamiento de productores de alto costo por otros de bajo costo, caso en el cual se produce un incremento del bienestar, tanto en la región integrada como en el mundo. La desviación de comercio implica la sustitución de productores de bajo costo por otros de alto costo, lo que produce una pérdida del bienestar tanto en el ámbito regional como mundial.

Richard Lipsey y F. Gehrels introdujeron algunas modificaciones en las ideas vinerianas, cuando criticaron su excesivo énfasis en los efectos sobre la producción originados por las uniones aduaneras. De acuerdo con Lipsey, estas últimas producen una modificación en los precios relativos en los mercados de los países miembros, lo que, a su vez, tiene dos efectos importantes. El primero es un cambio en la localización de la producción, como fue analizado de forma amplia por Viner. El segundo es un efecto sobre el consumo, que origina una sustitución de mercancías y produce un cambio en el volumen del comercio ya existente, gracias a los bienes más baratos que se adquieren del nuevo socio comercial. Tal situación provocaría un incremento del volumen de las importaciones desde el país socio y, al mismo tiempo, una disminución de las importaciones del resto del mundo. ${ }^{4}$ La integración económica tendría un efecto sobre la producción y también sobre el consumo producido por la modificación de los precios relativos, aspecto no incluido en el análisis de Viner. Sin embargo, el mismo Lipsey criticó luego esta distinción entre efectos sobre la producción y sobre el consumo, por su excesivo énfasis en estos últimos y porque soslaya que los cambios en el consumo causan también un cambio en la producción. Lipsey propuso como categorías más exactas las de sustitución entre países y sustitución de mercancías. La primera comprende la creación y desviación de comercio vinerianas, es decir, cuando un país es sustituido por otro como proveedor de ciertos bienes. La segunda ocurre cuando un bien es sustituido por

3 Jacob Viner, The Customs Union Issue, Nueva York, Carnigie Endwment for Peace, 1950.

4 Richard Lipsey, "The Theory of Customs Unions/A General Survey", The Economic Journal, vol. LXX, núm. 270, febrero de 1960, pp. 40-46. 
otra mercancía, al menos parcialmente, como resultado del cambio de los precios relativos. ${ }^{5}$

El modelo dirigista concibe a la integración como un proceso que trasciende lo comercial y promueve el desarrollo económico integral y, en especial, la industrialización. Harry Johnson presentó los primeros aportes para superar la estrecha visión comercialista de la integración. De acuerdo con Johnson, la decisión de un Estado de favorecer una unión aduanera debe ser analizada en el contexto de su interés en promover bienes públicos, lo que supone que existe una preferencia colectiva favorable al desarrollo industrial. ${ }^{6}$ Johnson rechaza el supuesto de que los aranceles sólo se utilizan para promover objetivos económicos, ya que también tienen propósitos políticos. De acuerdo con estas premisas, Johnson argumenta que las uniones aduaneras se crean básicamente para proteger "una preferencia colectiva por el sector industrial". La integración se considera como un mecanismo eficiente para estimular las inversiones dirigidas a promover nuevos desarrollos industriales. Además, la integración facilitaría la realización de otros objetivos económicos, pues gracias a la industrialización los países podrían mejorar sus términos de intercambio con el resto del mundo e incrementar su poder de negociación vis-à-vis otros más grandes. ${ }^{7}$

El otro enfoque dirigista importante es el representado por el pensamiento estructuralista. Éste concibe que el objetivo de la integración no es fusionar mercados sino economías mediante la unión progresiva de los espacios económicos nacionales en una nueva entidad económica y política, caracterizada por la solidaridad entre sus miembros. ${ }^{8}$ Este tipo de integración sólo puede emprenderse entre países vecinos geográficamente y con similares niveles de desarrollo económico, mediante la adopción de un conjunto de medidas que faciliten la producción en gran escala, permitan diversificar la producción y promuevan el progreso técnico. De acuerdo con este enfoque, la integración descansa no sólo en los mecanismos de mercado sino que requiere una participación del Estado como director del proceso.

El enfoque estructuralista no concibe la economía nacional como un conjunto homogéneo, armónico y equilibrado, sino complejo, heterogéneo y estructu-

5 Ibid.

6 Harry Johnson, "An Economic Theory of Protectionism, Tariff Bargaining and the Formation of Customs Unions", Journal of Political Economy, núm. 72, 1965, p. 27.

7 Joseph Brada y José Méndez, "Political and Economic Factors in Regional Economic Integration”, Kyklos, vol. 46, núm. 2, 1993, p. 187.

8 André Marchal, Integración y regionalización de la economía europea, Madrid, Seminarios y Ediciones, S.A., 1970, p. 124. 
rado, demasiado alejado de la visión liberal basada en la teoría del equilibrio y su restablecimiento automático. Por ello, el Estado debe encauzar un proceso continuado, socialmente equilibrado y armonioso mediante políticas dirigistas. Como señala Vacchino:" "tal política puede valerse del mercado y sus automatismos, pero, también y principalmente, debe valerse de las acciones deliberadas y de las instituciones". La integración debe hacer corresponder las políticas de mercado con las dirigidas por los estados y las instituciones "de modo que sea factible eliminar las discriminaciones nacionales y originar un mercado unificado que, entre otras cosas, proporcione las directrices del proceso de desarrollo económico y social comunitario". ${ }^{10}$

Existieron variantes intervencionistas en las experiencias de integración en Europa Occidental y en América Latina. En esta última región fue importante la influencia del modelo de integración propuesto por la CEPAL durante las décadas de 1960 y 1970, que promovía la industrialización a escala regional. La integración era concebida como un mecanismo para hacer avanzar los programas de desarrollo industrial que algunos países habían comenzado a desarrollar en décadas anteriores y que peligraban de continuar en su fase de producción de bienes de capital, debido a la estrechez de los mercados nacionales. La continuación de la industrialización en el ámbito regional, permitiría crear fábricas con un tamaño adecuado para aprovechar la reducción de los costos medios derivados de las economías de escala, al incrementar su eficiencia y evitar que existiese una capacidad instalada ociosa. De igual manera, permitiría financiar de forma conjunta programas industriales que requerían ingentes inversiones en capital y tecnologías que no existían en la mayoría de los países de la región. Esto requería una fuerte participación del Estado en la promoción de las nuevas industrias y una política de protección comercial frente a terceros. En la propuesta de la CEPAL, durante la primera fase de la integración, los bienes de las nuevas industrias estarían sometidos a un régimen de libre comercio entre los países miembros, lo que crearía un mercado regional en el que las nuevas producciones aprenderían a ser competitivas, antes de ser sometidas a la competencia extrarregional. ${ }^{11}$ Éste fue el modelo que inspiró la primera etapa de la integración en América Central y la propuesta de crear un mercado común latinoamericano. Sin embargo, por diversas razones la integración adquirió un sesgo comercialista a partir de 1960 y la propuesta

9 Juan Mario Vacchino, op. cit., p. 166.

10 Ibid., p. 162.

11 Sobre el modelo cepalino de la integración, véase CEPAL, El Mercado Común Latinoamericano, Santiago de Chile, 1958. 
del mercado común terminó convirtiéndose en una de apertura comercial cuya expresión fue la Asociación Latinoamericana de Libre Comercio (Alalc). ${ }^{12}$

La integración europea puede también ser descrita como intervencionista, aunque de una forma diferente que en América Latina porque el objetivo de la Comunidad Económica Europea, actualmente Unión Europea, no fue la industrialización, ya avanzada en el viejo continente. Sin embargo, en la integración europea se han establecido políticas de protección a los consumidores, de apoyo a las regiones menos favorecidas de los países miembros y sobre todo la política agrícola común, que han supuesto una significativa intervención del Estado. Las ideas de Thomas Biersteker ${ }^{13}$ sobre el papel del Estado en la economía permiten validar el argumento sobre el carácter intervencionista del modelo de integración en Europa. Según Biersteker es posible encontrar al menos seis modalidades de intervencionismo estatal en la economía:

1) La primera modalidad se propone influir de manera positiva en la conducta de los empresarios a través de la política fiscal o la política monetaria.

2) La segunda regula la conducta de los actores económicos privados o canaliza su actividad económica mediante políticas para proteger el medio ambiente, los programas de seguridad social o la legislación del trabajo.

3) Una tercera modalidad de intervención busca transformar al Estado en mediador entre el capital y el trabajo.

4) La cuarta modalidad es la redistribución del producto social y privado mediante subvenciones o por la política industrial.

5) La quinta es la producción directa de bienes y de servicios.

6) Finalmente, la última modalidad de intervención del Estado en la economía es la planificación. ${ }^{14}$

Con la aceptación de las ideas de Biersteker, es fácil observar varios tipos de políticas intervencionistas en la integración europea. Por ejemplo, la legislación comunitaria de protección del medio ambiente o de defensa de los consu-

12 Sobre este tema, véase José Briceño Ruiz, "El viejo y el nuevo regionalismo caribeño. Un análisis comparado de la teoría y práctica de las experiencias de integración en las américas”, en José Briceño Ruiz (compilador), Escenarios de la integración regional en la Cuenca del Caribe, Mérida, Venezuela, Consejo de Publicaciones de la Universidad de los Andes, 1999; y Germánico Salgado, "El mercado regional latinoamericano: el proyecto y la realidad", Revista de la CEPAL, Santiago de Chile, abril de 1979, pp. 87-133.

13 Thomas Biersteker, "Reducing the Role of the State in the Economy: The Politics of Stabilization and Structural Adjustment", ponencia presentada en la Reunión Anual de la America Political Science Association, Washington, D.C., agosto de 1988.

14 Ibid. 
midores es sin duda una modalidad de intervencionismo regulador. La política agrícola común o la política de apoyo a las regiones menos desarrolladas es una forma de intervencionismo redistribuidor. El proyecto de construcción del Airbus es un ejemplo de intervencionismo cuyo objetivo es la producción de un bien en particular. En consecuencia, es válido describir a la experiencia de integración en Europa Occidental como intervencionista.

El regionalismo abierto en América Latina también puede ser concebido como variante intervencionista. Existe una tendencia a confundir el regionalismo abierto con la mera apertura comercial promovida en los programas de ajuste estructural. Según la CEPAL, en los años recientes en América Latina y el Caribe se han desarrollado dos modalidades de integración, una producto de los acuerdos comerciales intergubernamentales que promueven la desgravación arancelaria, descrita como integración impulsadas por políticas, y otra descrita como integración de hecho, resultado de las políticas macroeconómicas y comerciales que han implementado los Estados de la región. ${ }^{15}$ La CEPAL describe el regionalismo abierto como "el proceso que surge de conciliar [...] la interdependencia nacida de los acuerdos especiales de carácter preferencial y aquella impulsada básicamente por las señales del mercado resultantes de la liberalización comercial en general". ${ }^{16}$ En el modelo del regionalismo abierto, la integración es concebida como un complemento de las políticas aperturistas dirigidas a mejorar la inserción de los países latinoamericanos en la economía mundial, que deja de lado las viejas políticas de crecimiento hacia adentro. En otras palabras, "el regionalismo abierto puede ser entendido como un camino 'no multilateral' para avanzar hacia un sistema internacional de comercio más abierto. Esta visión del regionalismo puede interpretarse como aquella integración que no constituye murallas ni tiende a convertirse en una fortaleza aislada del resto del mundo". ${ }^{17} \mathrm{Si}$ el análisis se limita a este aspecto de la apertura, es cierto que se podrían encontrar similitudes con las propuestas aperturistas de inspiración neoliberal propias del regionalismo abierto en Asia y en el Pacífico. Si éste fuera el caso, la versión latinoamericana del regionalismo no tendría nada de original. Ésta es la opinión de autores como Salgado, ${ }^{18}$ para

15 CEPAL, El regionalismo abierto en América Latina y el Caribe. La integración al servicio de la transformación productiva con equidad, Santiago de Chile, CEPAL, 1994.

16 Ibid., p. 13.

17 Andrés Cisneros y Jorge Campell, "El Mercosur: regionalismo abierto o un Building Block", Boletim de Integração Latino-Americana [en línea], núm. 19, Brasilia, julio-diciembre de 1996, <http://www.mre.gov.bt/getec/WEBGETEC/BILA>, consultada el 6 de noviembre de 2000.

18 Germánico Salgado, "El Grupo Andino: entre dos concepciones de la integración económica", Síntesis, núm. 24, Madrid, julio-diciembre de 1994, p. 84. 
quien las similitudes del regionalismo abierto con el comercialismo neoliberal son considerables. Por ello, podría ser percibido como un simple regreso a la teoría vineriana de las uniones aduaneras, que considera a la integración como un subóptimo en el camino hacia la total liberalización del comercio multilateral.

Sin embargo, la versión cepalina del regionalismo abierto supera la simple liberalización comercial, aunque éste sea el elemento que más se conoce de la propuesta. Un elemento realmente innovador del regionalismo abierto es su intento de incorporar la propuesta de una transformación productiva con equidad dentro de la política de integración. En América Latina se requiere un incremento de la productividad mediante un proceso de absorción y difusión del proceso técnico adquirido, el aumento del tamaño de los mercados regionales, la sinergia entre empresa, sectores e instituciones y, en términos más generales, por la eficiencia sistema de la estructura productiva. ${ }^{19}$ Para la CEPAL, la integración podría colaborar en este proceso de transformación productiva e incorporación y difusión de las tecnologías disponibles en el mundo. La ampliación de los mercados y el incremento de las vinculaciones entre empresas, sectores e instituciones a escala subregional, permiten el desarrollo de acciones conjuntas para impulsar el desarrollo gradual de ventajas comparativas en sectores en los cuales el desarrollo tecnológico y el aprendizaje sean facilitados por la acción regional. De igual manera, la integración contribuiría a incrementar la eficiencia de las industrias sustitutivas de importaciones, que ante la eliminación de las distorsiones generadas por el nivel y estructura del sistema tradicional de protección, podrían convertirse en industrias exportadoras y utilizar al mercado regional como fase previa antes de su exposición plena a la competencia global. Asimismo, la integración puede ser útil en el fomento de la innovación tecnológica. Los gustos similares de la población y la cercanía geográfica permitirían usar al mercado regional como el espacio en el cual se explotasen las innovaciones tecnológicas en los nuevos productos al menor costo y con menor grado de incertidumbre que en los mercados extrarregionales. La integración también puede incrementar el poder de negociación conjunto de la región y realizar mejoras en la infraestructura y en la facilitación del comercio, elementos que coadyuvan a la transformación productiva. ${ }^{20}$ Todo esto demuestra que el regionalismo abierto de la CEPAL es algo más que una

19 Comisión Económica para América Latina (CEPAL), Transformación productiva con equidad, Santiago de Chile, Naciones Unidas, 1990, p. 166.

20 Ibid., pp. 166-170. 
propuesta de apertura comercial ya que se articula perfectamente con la tradición de las ideas prebischianas sobre el papel de la integración en el cambio estructural de las economías latinoamericanas.

\section{EL TLCAN COMO UN NUEVO MODELO DE INTEGRACIÓN}

$\mathrm{El}$ análisis de las diversas teorías hace difícil categorizar al TLCAN como modelo de integración, ya que éste no constituye una modalidad de integración dirigista, pues ha excluido cualquier dispositivo de planificación del desarrollo, programación industrial o cualquier mecanismo para mitigar las asimetrías resultantes de los diversos niveles de desarrollo de Canadá, Estados Unidos y México. Esto podría llevar a inferir que el enfoque comercialista inspira al TLCAN, si tomamos en cuenta que éste se propone establecer una zona de libre comercio en 15 años, la liberalización del movimiento de capitales y la protección de las inversiones. Sin embargo, un análisis más cuidadoso conduce a concluir que el TLCAN se aleja en varios aspectos de los modelos comercialistas tradicionales, ya que se propone la meta del libre comercio sin aspirar a trascender automáticamente a fases superiores de integración, como la unión aduanera o el mercado común. Esto fue expresamente rechazado por el gobierno de Estados Unidos debido a presiones de diversos grupos políticos y económicosociales, que se oponen a la creación de una unión aduanera o, como es el caso de los trabajadores, quienes rechazan la libre circulación de la mano de obra. ${ }^{21}$ Esto per se no es lo realmente innovador. Otros esquemas comercialistas de integración como la Asociación Europea de Libre Comercio (EFTA por sus siglas en inglés) o la Asociación de Libre Comercio del Caribe (Carifta) tampoco se propusieron superar el libre comercio. Lo particular del TLCAN es que al tímido enfoque de una zona de libre comercio se unen ambiciosos objetivos económicos, como la regulación de sectores vitales de las economías de los países miembros. Esto convierte al TLCAN en un esquema que trasciende las medidas convencionales de eliminación de las restricciones arancelarias. ${ }^{22}$

$\mathrm{El}$ acuerdo regula el sector comercio, los servicios, las inversiones, la propiedad intelectual, las compras gubernamentales, mientras los acuerdos paralelos en materia laboral y ambiental reglamentan dos temas crecientemente vinculados con el comercio. ${ }^{23}$ Muchos de estos aspectos no eran incluidos en los tradicionales proyectos de integración comercialista. Incluso en materia de

21 Björn Hettne y Edmé Dominguez, "In the European Footsteps: NAFTA as a Case of Regionalism”, en Weine Karlsson y Axhil Malaki (editores), Growth, Trade and Integration in Latin America, Estocolmo, Institute of Latin American Studies, 1996, p. 175.

22 Hans Joachin Lauth, "NAFTA y su importancia para América Latina: ¿mayor vinculación o factor de separación", en Manfred Mols et al., Cambio de paradigmas en América Latina, nuevos impulsos, nuevos temores, Caracas, Nueva Sociedad, 1994, p. 166.

23 Ibid. 
liberalización comercial, el TLCAN presenta innovaciones que permiten considerarlo como único en su tipo. ${ }^{24}$ Su enfoque es amplio y comprensivo, incluyendo a todos los sectores de la economía en el programa de liberalización comercial, incluso la agricultura, un sector tradicionalmente sometido a un tratamiento especial en los esquemas de integración comercialista.

Uno de los aspectos más innovadores del TLCAN son las medidas relacionadas con las inversiones. Aunque este aspecto ha sido regulado en otros esquemas de integración, en el TLCAN destaca el relajamiento de estándares internacionales sobre la materia y el excesivo apoyo al capital extranjero. En el TLCAN se establece que ningún gobierno local o nacional puede regular o influir sobre cualquier inversión que esté relacionada con el comercio. Igualmente no se permite ningún trato especial a las empresas locales o nacionales en relación con el dado a las de los socios del TLCAN, que reciben un tratamiento nacional. En consecuencia, se prohíbe a los gobiernos que obliguen a los inversionistas a adquirir materias primas de fuentes locales, reinvertir parte de las ganancias en el país receptor o medidas similares. Esto también se expresa en la inclusión de la propiedad intelectual como parte del acuerdo, un hecho casi inédito en el viejo comercialismo.

Esta modalidad de integración obedece en gran medida a la dinámica política subyacente en el TLCAN, que constituye una estrategia de integración regional que es producto de las presiones de ciertos actores económicos, en particular las empresas trasnacionales (ETN) y nacionales, que buscan crear un entorno favorable para sus actividades en un contexto internacional crecientemente globalizado. En un mundo posfordista, las ETN han descubierto la validez de la integración regional. La importancia de la geografía entre usuarios y proveedores y la necesidad de obtener una reducción de los costos del factor trabajo explican este interés en el regionalismo económico. Las ETN prefieren localizar su producción en países de bajo costo que sean cercanos a los centros de decisión, con el objetivo de facilitar una rápida adaptabilidad a los cambios de la demanda y mantener flujos adecuados de información e innovación tecnológica. ${ }^{25}$ Estas fuerzas trasnacionales ejercen su lobby sobre las instancias de poder político para imponer un modelo de integración favorable a la expansión de sus actividades y para que se establezca un marco de seguridad jurídica amplio. Este análisis sugiere que las ETN estadounidenses son las principales impulsoras

24 Emilio Caballero, El Tratado de Libre Comercio, México, UnAM/Diana, 1991, p. 42.

25 Carlos J. Moneta, "Espacios económicos e inserción externa: nuevos parámetros", Capítulos SELA, núm. 50, abril-junio de 1997, p. 38. 
del modelo de integración del TLCAN, pues, mediante la inclusión de México, obtienen una posición privilegiada en un mercado cercano y de mano de obra barata y les permite mantener o incrementar su competitividad. La importancia de las ETN en el diseño del TLCAN explica el carácter librecambista del acuerdo, la trascendencia otorgada a la protección de las inversiones y la propiedad intelectual y la creación de instancias intergubernamentales para la solución de conflictos, medidas que facilitan las actividades de las ETN.

Sin embargo, los empresarios nacionales también apoyan la nueva lógica de la integración. Por ejemplo, el establecimiento de programas de ajuste estructural de las economías ha supuesto la eliminación de las viejas políticas de proteccionismo que permitieron el crecimiento de muchas empresas en México. Este nuevo contexto económico obliga al empresariado mexicano a insertarse en nuevos mercados y a ser más competitivo. Una integración económica que favorezca este objetivo mediante la liberalización del comercio y de las inversiones es apoyada por el empresariado mexicano. Este protagonismo de las ETN y de los empresarios nacionales no supone que los estados han sido totalmente excluidos del proceso. Contrariamente, han desempeñado un rol protagónico en la promoción de un modelo que reivindica a escala regional políticas económicas establecidas en el ámbito nacional. La mano visible del Estado está presente en el TLCAN, aunque éste se oriente fundamentalmente por la mano invisible del mercado. Sin embargo, la actividad estatal no es la de promotor o director del proceso, sino que se limita a establecer un marco jurídico-institucional que facilite el desarrollo del modelo de integración.

Las negociaciones de la Ronda Uruguay del Acuerdo General sobre Aranceles y Comercio (GATT) y el creciente proteccionismo en otros países desarrollados fueron dos factores que incidieron en la decisión de los estados nacionales en América del Norte de apoyar el modelo TLCAN. Estados Unidos dejó de ser el hegemon de la economía mundial y sus posiciones en diversos temas no fueron aceptadas por sus socios, como ocurrió en las negociaciones de la Ronda Uruguay del GATT con relación a la propiedad intelectual, la protección de las inversiones o la agricultura. La integración regional permite a Estados Unidos regular en un ámbito más limitado tales temas, además de tener la ventaja adicional de enfrentarse a países que tienen una capacidad económica infinitamente inferior a la suya. ${ }^{26}$ De esta manera, la integración se convierte en un elemento de disuasión frente a los países que rechacen ciertas posiciones esta-

26 María Cristina Rosas, "Crisis del multilateralismo clásico: política comercial externa de Estados Unidos y zonas de libre comercio”, Revista Mexicana de Ciencias Políticas y Sociales, vol. xxxix, núm. 157, nueva época, México, julio-septiembre, 1994, p. 46. 
dounidenses en materia de comercio e inversiones en las negociaciones multilaterales.

El temor de una crisis del régimen multilateral también creó condiciones para que la integración regional fuera aceptada en Canadá y México. Para ambos países la integración con Estados Unidos significa un acceso seguro a este mercado y la protección de cualquier acción proteccionista unilateral de Washington. Esto fue asegurado por Canadá en 1998 al firmar el acuerdo de libre comercio bilateral con Estados Unidos. Su interés en el TLCAN es mantener los privilegios obtenidos en tal acuerdo, que podrían ser afectados por las concesiones otorgadas a México. ${ }^{27}$ Este último, por su parte, considera al TLCAN vital para continuar su estrategia económica aperturista iniciada a mediados de los años ochenta. Esta estrategia tiene como uno de sus pilares la exportación de manufacturas, cuyo mayor destino es Estados Unidos, y la recepción de las inversiones extranjeras. El riesgo del proteccionismo unilateral estadounidense puede provocar el derrumbe de esta estrategia, por lo que el TLCAN se presentaría como un seguro. ${ }^{28}$

El TLCAN puede ser concebido como un nuevo modelo de integración, que es denominado en este ensayo comercialismo modificado. Su objeto central es la liberalización comercial, pero para ello no sólo se plantea la reducción de los aranceles sino también la creación de un ámbito jurídico-institucional favorable a tal objetivo. Por ello, regula aspectos como la propiedad intelectual y las inversiones. Este diseño económico tiene el apoyo político de las ETN, a su vez constreñidas por el nuevo escenario económico globalizado, y por amplios sectores del empresariado nacional y los estados, ante la imperiosa necesidad de enfrentar las dificultades del régimen de comercio multilateral, ampliar a un ámbito regional las políticas neoliberales y enfrentar problemas comunes. Sobre la base de este modelo, América del Norte desarrolla lo que algunos denominan una alianza estratégica para avanzar de la integración silenciosa a la integración formal. ${ }^{29}$

27 Ronald Wonnacott, "Canadá ante las negociaciones sobre el libre comercio entre México y Estados Unidos”, en Gustavo Vega Cánovas (coordinador), México ante el libre comercio con América del Norte, México, El Colegio de México/Universidad Tecnológica de México, 1992, p. 355. 28 Véase Nora Lustig, "NAFTA: Potential Impact on Mexico's Economy and Beyond", en Roberto Bouzas y Jaime Ros (editores), Economic Integration in the Western Hemisphere, Notre Dame, Notre Dame University Press, 1994, pp. 46-57; Silvia Ostry, "The NAFTA: its International Background", en Stephen Randall et al., North America without Borders, Integrating Canada, The United States and Mexico, Calgary, University of Calgary Press, 1992, pp. 27-28.

29 Lorraine Eden y Mauren Appel Molet, "De la integración silenciosa a la alianza estratégica: la economía política de libre comercio en América del Norte", en Gustavo Vega Cánovas (editor), Liberación comercial y libre comercio en América del Norte, México, El Colegio de México, 1993 , p. 25 


\section{¿QUÉ MODELO DE INTEGRACIÓN PARA EL ALCA?}

La propuesta de George Bush de establecer un área de libre comercio desde Alaska hasta Tierra del Fuego, anunciada en 1991 en el discurso de la Iniciativa para las Américas, inició un debate sobre el modelo de integración que se debe adoptar en el hemisferio occidental y sobre la conveniencia de transplantar el TLCAN al resto de América Latina y el Caribe. Aunque Bush no señaló en forma expresa que su propuesta de un área de libre comercio desde Alaska hasta la Patagonia debía inspirarse en el TLCAN, sus planteamientos así lo sugieren. Su insistencia sobre la necesidad de reformar el marco legal de las inversiones, de proteger la propiedad intelectual, de establecer una política comercial aperturista, evidenciaba el intento de ampliar el modelo TLCAN al resto del hemisferio. En principio, Estados Unidos pretendía negociar veinte o más tratados de libre comercio (TLC) con los países latinoamericanos y caribeños, en los que, mutatis mutandi, se adoptarían normas similares a las del acuerdo suscrito con México. Este proyecto hemisférico fue descrito por Richard Lipsey ${ }^{30}$ y Ronald Wonnacott ${ }^{31}$ como de ejes y radios. Mientras que Estados Unidos actuaría como el eje que tendría acceso al mercado de cada uno de los radios, los radios serían los países latinoamericanos y caribeños, que podrían ingresar al mercado estadounidense, pero estarían desconectados entre sí. En cada uno de los acuerdos de este sistema de ejes y radios, Estados Unidos buscaría imponer un modelo de integración aperturista y librecambista como el del TLCAN.

México favorece el modelo TLCAN, aunque teme que su ampliación al resto del hemisferio produzca la pérdida de su posición privilegiada en el mercado estadounidense. México ha desarrollado una diplomacia relativamente de bajo perfil, dirigida a salvaguardar en la medida de lo posible lo ganado en el TLCAN, pero que apoya al mismo tiempo las propuestas de Estados Unidos. El apoyo que México brinda al modelo TLCAN obedece a dos razones. Por una parte, la puesta en marcha del TLCAN supone un realineamiento estratégico de México. ${ }^{32}$ Como señala Chanona Burguete, el TLCAN representa el tránsito

30 Richard Lipsey, "Growth, Erosion and Restructuring of the Multilateral Trade System", Paper delivered at the Annual Meeting of the American Economic Association, Atlanta, Georgia, diciembre de 1989.

31 Ronald Wonnacott, "US Hub and Spoke Bilateral and Multilateral Trading System", $C D$ Howe Commentary, núm. 23, Toronto, octubre de 1990.

32 José Briceño Ruiz, Del Mercado Común Latinoamericano al Área de Libre Comercio de las Américas. Las posibilidades de convergencia entre la integración latinoamericana y caribeña en la era de la globalización, Documento de Trabajo, núm. 005, Área de Relaciones Internacionales y Globales/CEVERIG, Universidad Central de Venezuela, 1998. 
desde una integración económica informal a una integración económica formal, cuyo resultado es el establecimiento de una alianza estratégica entre ambos países. ${ }^{33}$ Quiérase o no, esto ha conducido a un mayor alineamiento de México con Estados Unidos en diversos temas políticos y económicos, por lo que se puede afirmar, como hace Chabat, que en la década de 1990, México está "tan cerca de Estados Unidos y tan lejos de América Latina”. ${ }^{34} \mathrm{El}$ segundo factor es que México ha desarrollado su propia estrategia de ejes y radios en la Cuenca del Caribe a través de la suscripción de acuerdos sobre comercio e inversión con los países centroamericanos y con sus dos socios del Grupo de los Tres (Colombia y Venezuela). En estos acuerdos, México ha tenido éxito en promover el modelo TLCAN en lo que respecta a la liberalización comercial, al incluir la eliminación de las barreras no arancelarias, la regulación de las inversiones, la flexibilización de las normas sobre propiedad intelectual relacionadas con el comercio, la apertura de las compras gubernamentales a los inversionistas de los países socios, etc. Para especialistas como Valladão, ${ }^{35}$ esta estrategia tiene como propósito rodear al Mercosur con una serie de acuerdos de tipo TLCAN y mostrar la fuerza de México frente al bloque sudamericano dirigido por Brasil.

La estrategia de los países de la Cuenca del Caribe se acerca a la mexicana, aunque por razones diferentes. La Comunidad del Caribe (Caricom) y el Mercado Común Centroamericano (MCCA) han apoyado el modelo TLCAN debido a su alta dependencia del mercado de Estados Unidos. La no participación en el ALCA podría tener un elevado costo en términos de un relativo aislamiento de estas pequeñas economías, la reducción de su atractivo por las inversiones extranjeras y su mayor marginalización a escala hemisférica. El establecimiento del TLCAN ya ha tenido un impacto negativo sobre las economías caribeñas. La presencia mexicana en el TLCAN ha producido una disminución de las preferencias comerciales de la Iniciativa de la Cuenca del Caribe. Debido a su cercanía al mercado de Estados Unidos y Canadá, el bajo costo de su mano de obra y la reducción arancelaria prevista en el TLCAN, México ha desplazado a varios países caribeños en el mercado estadounidense. De igual manera, México los ha sustituido como destino de inversiones estadounidenses que buscan bajos costos de producción. En este contexto, es comprensible que los países caribeños no intenten promover posiciones de negociación contrarias a

33 Chanona Burguete, op. cit., p. 83.

34 Jorge Chabat, "Mexico: So Close the United States so Far from Latin America", Current History, vol. 92, núm. 571, febrero de 1993, pp. 55-58.

35 Alfredo G. A. Valladão, Le Retour du Panaméricanisme. La Stratégie des Etats Unis en Amérique Latine après la guerre froide, París, CREST, 1994. 
Estados Unidos, su socio comercial más importantes en la región. La única reivindicación caribeña, contraria al modelo TLCAN, es su reconocimiento como economías pequeñas y menos desarrolladas que necesitan un tratamiento preferencial. Al margen de esto, los países de la Caricom y del MCCA apoyan in grosso modo la mayoría de las políticas del modelo TLCAN. La nueva etapa de la integración de la Caricom y del MCCA se ha caracterizado por su adaptación a la lógica de apertura mediante la eliminación de las políticas de programación industrial y de protección arancelaria que caracterizaron a la integración en las décadas pasadas. Estas políticas han sido sustituidas por mecanismos de mercado y por la inclusión en el proceso de integración de temas como la propiedad intelectual, los servicios y las compras gubernamentales, como en el TLCAN.

El caso del Mercosur es diferente, ya que éste "es una estrategia para aumentar la competitividad de los países miembros en la economía mundial". ${ }^{36}$ Este esquema de integración ha desarrollado una diplomacia para fortalecer la cohesión regional, mediante la creación de un mercado ampliado, diversificar la estructura productiva y promover la industrialización, impulsar el cambio tecnológico y generar nuevas ventajas competitivas. El Mercosur no es una simple estrategia aperturista que busca ataer a las ETN y aprovechar su interés en formas de producción regional, sino una respuesta a la globalización que no invalida los procesos endógenos de crecimiento, cohesión regional y transformación productiva.

En el Mercosur, se rechaza el tipo de apertura realizada en el TLCAN, con exclusión de la liberalización indiscriminada del sector servicios y las compras gubernamentales y cualquier regulación de la propiedad intelectual que exceda lo acordado en la Organización Mundial del Comercio (OMC). Por ejemplo, se opone a cualquier apertura radical de las telecomunicaciones, los servicios financieros y los programas de compras gubernamentales como se ha realizado en el TLCAN. Eso sería contrario al Protocolo de Colonia que permite a los estados reservar a ciudadanos del el Mercosur las operaciones de los sistemas telefónicos, la propiedad de medios de comunicación, la participación en servicios de intermediación financiera y las ofertas de contratos sobre adquisiciones oficiales. ${ }^{37}$ En otras palabras, el Mercosur está promoviendo un modeo de integración más intervencionista, en el que el libre comercio se combina con la promoción industrial y el apoyo a la renovación tecnológica.

36 Aldo Ferrer, "Nuevo escenario internacional. Los dilemas del Mercosur", Encrucijadas / Revista de la Universidad de Buenos Aires, año 2, núm. 1, abril de 1995, p. 34.

37 Informe Latinoamericano, 5 de agosto de 1997, p. 364. 
Existen factores, que van más allá de la simple racionalidad económica, que explican la reticencia del Mercosur en relación con el modelo TLCAN. El Mercosur se ha ganado el prestigio de ser el esquema de integración más exitoso de América Latina y el Caribe, y según un especialista ruso: "El Mercosur se ha granjeado el reconocimiento internacional en tanto que ente soberano en el ámbito mundial y ha atesorado experiencia relacionada con la concertación de importantes acuerdos entre bloques." ${ }^{38}$ Esto significa que el Mercosur ha adquirido ya un peso específico en la política internacional, y sus países miembros, en particular Brasil, no pueden aceptar su desaparición o subordinación al proyecto hemisférico.

Chile, una de las economías líderes en América Latina, miembro asociado del Mercosur y el segundo convidado a ingresar al TLCAN, también tiene un enfoque sobre la integración regional algo distinto al de Estados Unidos. Aunque el ingreso al mercado estadounidense constituyó una prioridad para Chile, hasta el inicio de las negociaciones de su adhesión, a partir de 1997, se produjo un cambio en las prioridades en la política comercial externa chilena hacia el Mercosur. Es cierto que este cambio en la posición chilena fue resultado de las dificultades del gobierno de Clinton para obtener un nuevo Fast Track, pero también evidencia las dudas de Chile con respecto al modelo de integración que promueve Estados Unidos. Chile tiene ya una experiencia negociadora de acuerdos comerciales, en los que ha obtenido ventajas que perdería si adopta el modelo TLCAN. El acuerdo de libre comercio entre Chile y Canadá demuestra cuál es el tipo de integración que apoya Chile y sus diferencias con el TLCAN. En el acuerdo chileno-canadiense se mantuvieron las restricciones a las inversiones de corto plazo, algo que Estados Unidos considera como una discriminación a la inversión extranjera, contraria al espíritu del TLCAN. También se estableció un sistema de normas de origen que exigen $35 \%$ de contenido local, monto significativamente inferior al $60 \%$ requerido en el TLCAN. A diferencia de este último, el acuerdo no prevé la liberalización de las inversiones en los servicios financieros, no regula la propiedad intelectual y obliga a los países a eliminar los gravámenes antidumping. ${ }^{39} \mathrm{El}$ inicio en diciembre de 2000 de negociaciones bilaterales para crear una zona de libre comercio entre Chile y Estados Unidos, significó un regreso de Chile a su estrategia de conquista del mercado del norte. Resta esperar cuál modelo de integración se

38 Anatoly Glinkin, "NAFTA y Mercosur en el camino del siglo XXI: búsqueda de un compromiso", Revista Iberoamericana [en línea], núm. 1, 1998, Academia de Ciencias de Rusia, Instituto de Latinoamérica, <hhtp://www.plugcom.ru/ ilaran/revista>, consultado el 1o. de marzo de 2000. 39 Informe Latinoamericano, 5 de agosto de 1997, p. 364. 
adoptará en el acuerdo y si Chile podrá obtener concesiones similares a las existentes en el acuerdo con Canadá.

Es evidente entonces que no existe un consenso sobre el modelo de integración que se debe llevar a cabo en las Américas. El proyecto estadounidense de ampliar el TLCAN en un sistema de ejes y radios fracasó debido a su rechazo por parte de los demás países de la región, en particular por parte del Mercosur. Durante el periodo de prenegociación del ALCA, que se desarrolló entre la reunión ministerial de Denver en 1995 y la reunión ministerial de San José, Costa Rica, en 1998, fueron notorias las diferencias entre el Mercosur y los países del TLCAN, en particular Estados Unidos, en diversos temas como el acceso a los mercados, las inversiones, el comercio agrícola, las compras gubernamentales, las normas de origen, las normas laborales y ambientales, etc. De igual manera, existieron diferencias en cuanto a la naturaleza misma del proceso negociador, ya que el Mercosur apoyaba la gradualidad y el respeto de los acuerdos ya existentes, lo que permitiría al Mercosur consolidarse como grupo y evitar "encontrarse en la situación de un peso ligero enfrentado en el cuadrilátero con un peso pesado". ${ }^{40}$

Contrariamente a la imagen de una América Latina hipnotizada por la propuesta del ALCA, estudios económicos demuestran que no todos los países de la región tienen los mismos incentivos para negociar con Estados Unidos. Esto ha sido explicado de forma meridiana por especialistas como Roberto Bouzas, ${ }^{41}$ quien concluye que para los países de América del Sur, excepto Colombia y Venezuela, no existen elevados incentivos positivos para negociar el ALCA. Ciertamente, existen importantes incentivos negativos derivados del riesgo de exclusión del proceso o del costo a pagar en caso de un ingreso retardado. Sin embargo, ¿son estos incentivos negativos suficientemente elevados como para llevar a los países del Mercosur a aceptar el modelo TLCAN? La respuesta durante el periodo de prenegociación fue negativa, lo que creó un escenario de conflicto, que aunque fue resuelto durante la Cumbre de Santiago, reapareció durante la fase formal de negociación que se inició en 1998 y se evidenció con toda crudeza en la III Cumbre Hemisférica de Quebec, en abril de 2001.

Autores como Dabène señalan que más que una oposición, lo que existe entre el Mercosur y el TLCAN es una convergencia, producto del predominio en ambos esquemas de integración de las políticas del Consenso de Washington. Sin

40 Glinkin, op. cit.

41 Roberto Bouzas, "El regionalismo en el hemisferio occidental: el NAFTA, el Mercosur y después”, Desarrollo Económico, vol. 36, Buenos Aires, verano de 1996, pp. 87-108. 
embargo, el mismo autor reconoce que existen diferencias en diversas áreas como los servicios y la propiedad intelectual. ${ }^{42}$ La realidad es que a pesar de la convergencia de las políticas económicas en torno a las propuestas neoliberales, es obvio que existen matices. Brasil, por ejemplo, ha sido el país que ha aplicado con mayor gradualidad el ajuste. El Mercosur en general es un grupo en el que se han mezclado las propuestas neoliberales y ciertos mecanismos de intervención económica, por lo que Aldo Ferrer lo ha descrito como un proceso que ha vivido entre la integración sustentable y el Consenso de Washington. ${ }^{43}$ En consecuencia, el interés de una convergencia con el TLCAN ha existido cuando el Consenso de Washington ha sido predominante dentro del Mercosur, como por ejemplo en 1990, cuando Carlos Saul Menen y Fernando Collor de Melo suscribieron el Acta de Buenos Aires. Sin embargo, luego de la elección de Fernando Henrique Cardoso en Brasil y del éxito del Mercosur en establecer una zona de libre comercio y una unión aduanera imperfecta, la idea de una integración sustentable readquirió vigor. Como ella supone cierta forma de intervención del Estado, su oposición al comercialismo radical del TLCAN es innegable.

Finalmente, existe otro factor que debe analizarse al considerar el rechazo al proyecto de extender el modelo TLCAN al resto del continente americano: la crisis mexicana de 1994. Esta crisis significó el fin de la ilusión de que la reforma neoliberal era el camino seguro y sin contramarchas al desarrollo y al crecimiento económico. Aunque esta crisis no sea resultado directo del TLCAN o la importancia de éste en su detonación sea menor que la señalada por los grupos contrarios a la integración con Estados Unidos, fue percibida de alguna manera en el resto de América Latina como vinculada al TLCAN. No es cierto, como señala Wiarda, ${ }^{44}$ que en América Latina el TLCAN es considerado como la causa de los problemas económicos que México vivió luego de la crisis de 1994. Es verdad, en cambio, que la crisis ayudó a cambiar la percepción políticamente favorable hacia el TLCAN, al mostrar que la integración con Estados Unidos no era garantía de estabilidad.

42 Olivier Dabène, "L'Intégration Régionales dans les Amériques. Economie Politique de la Convergence", Les Etudes du CERI, núm. 45, París, septiembre de 1998, pp. 5-6.

43 Aldo Ferrer, "El Mercosur: entre el Consenso de Washington y la integración sustentable", Comercio Exterior, vol. 47, núm. 5, mayo de 1997, pp. 347-354.

44 Howard Wiarda, "Consensus Found, Consensus Lost: Disjunctures in US Policy Toward Latin America in the Turn of the Century", Journal of Interamerican Studies and World Affairs, vol. 39, núm. 1, primavera de 1997, p. 25. 


\section{CONCLUSIÓN}

El TLCAN supone la aparición de una nueva forma de comercialismo en que se radicaliza la apertura comercial y la flexibilización de las normas vinculadas con el comercio. Junto a este modelo persiste la vieja tradición intervencionista que se ha mantenido en la Unión Europea y en el Mercosur. Estos modelos diversos de integración se han confrontado durante el proceso negociador del ALCA. La Iniciativa para las Américas (IPA) era en principio un proyecto para extender el TLCAN, que tuvo una enorme receptividad en América Latina y el Caribe. Sin embargo, los miembros del Mercosur han optado por una estrategia de integración que se opone en diversos aspectos al modelo TLCAN. Estas diferencias se han manifestado durante el proceso de prenegociación que se inició luego de la cumbre de las Américas de Miami y ha reaparecido en la Cumbre de Quebec. Durante estas negociaciones los países del Mercosur han propuesto un modelo de integración que se opone al radicalismo del TLCAN en materias como la liberalización de las inversiones y la regulación de la propiedad intelectual. Este comercialismo radical del TLCAN no puede ser aceptado por todos los países de la región, pues al lado de este modelo existen otras variantes de integración que, sin excluir los mecanismos de mercado, otorgan aún un valor importante al Estado. Está claro que un TLCAN sin el Mercosur no es atractivo para Estados Unidos, pero es también obvio que como potencia hegemónica que es intentará imponer el modelo del ALCA. Y, parafraseando a Wiarda, como en Estados Unidos existe la tendencia a crear una dicotomía entre mercantilismo o libre mercado, la defensa del modelo TLCAN será percibida como una defensa al libre comercio, mientras que las exigencias latinoamericanas se percibirán como mercantilistas. El problema es que en las realidades del desarrollo latinoamericano las cosas no son tan claras. ${ }^{45}$ En consecuencia, es necesario que en Estados Unidos se reconozca que existen posiciones intermedias y una variedad de modelos intermedios, en los que América Latina tiene un cierto genio para improvisar. ${ }^{46} \mathrm{El}$ aceptar esto puede facilitar el proceso de negociación del ALCA.

\section{BIBLIOGRAFÍA}

- Biersteker, Thomas (1988), "Reducing the Role of the State in the Economy: The Politics of Stabilization and Structural Adjustment", ponencia

45 Ibid., p. 29.

46 Ibid. 
presentada en la reunión anual de la American Political Science Association, Washington, D.C., agosto.

- Brada, Joseph y José Méndez (1993), "Political and Economic Factors in Regional Economic Integration”, Kyklos, vol. 46, Facs. 2, 1993, pp. 183-201.

- Bouzas, Roberto (1996), "El regionalismo en el hemisferio occidental: el NAFTA, el Mercosur y después”, Desarrollo Económico, vol. 36, Buenos Aires, verano, pp. 87-108.

- Briceño Ruiz, José (1999), "El viejo y el nuevo regionalismo caribeño. Un análisis comparado de la teoría y práctica de las experiencias de integración en las Américas", en José Briceño Ruiz (compilador), Escenarios de la integración regional en la Cuenca del Caribe, Mérida, Venezuela, Consejo de Publicaciones de la Universidad de los Andes.

- Briceño Ruiz, José (1998), "Del Mercado Común Latinoamericano al Área de Libre Comercio de las Américas/Las posibilidades de convergencia entre la integración latinoamericana y caribeña en la era de la globalización”, Documento de Trabajo, núm. 005, Área de Relaciones Internacionales y Globales/CEverig, Universidad Central de Venezuela.

- Caballero, Emilio (1991), El Tratado de Libre Comercio, México, unam/Diana.

- Comisión Económica para América Latina (CEPAL) (1994), El regionalismo abierto en América Latina y el Caribe. La integración al servicio de la transformación productiva con equidad, Santiago de Chile, CEPAL.

- CEPAl (1990), Transformación productiva con equidad, Santiago de Chile, Naciones Unidas.

- _ (1958), El Mercado Común Latinoamericano, Santiago de Chile, CEPAL.

- Cisneros, Andrés y Jorge Campell (1996), "El Mercosur: regionalismo abierto o un Building Block", Boletim de Integração Latino-Americana [en línea], núm. 19, Brasilia, julio-diciembre, <http://www.mre.gov.bt/getec/wEBGETEC/ BILA $>$, consultada el 6 de noviembre de 2000.

- Chabat, Jorge (1993), "México: So Close the United States so Far from Latin America”, Current History, vol. 92, núm. 571. febrero, pp. 55-58.

- Chanona Burguette, Alejandro (1993), "Una reconsideración de las teorías de la integración y el problema de la integración económica formal entre México y Estados Unidos", Relaciones Internacionales, vol. Xv, núm. 60, nueva época, México, octubre-diciembre, pp. 87-94.

- Dabène, Olivier (1998), "L’Intégration Régionales dans les Amériques. Economie Politique de la Convergence", Les Études du CERI, núm. 45, París, septiembre. 
- Eden, Lorraine y Mauren Appel Molet (1993), "De la integración silenciosa a la alianza estratégica: la economía política de libre comercio en América del Norte", en Gustavo Vega Cánovas (editor), Liberación comercial y libre comercio en América del Norte, México, El Colegio de México.

- Ferrer, Aldo (1997), "El Mercosur entre el consenso de Washington y la integración sustentable", Comercio Exterior, vol. 47, núm. 5, México, mayo, pp. 347-354.

- (1995), "Nuevo escenario internacional. Los dilemas del Mercosur", Encrucijadas. Revista de la Universidad de Buenos Aires, año 2, núm. 1, abril, pp. 31-37.

- Glinkin, Anatoly (1998), "NAFTA y Mercosur en el camino del siglo Xxi: búsqueda de un Compromiso", Revista Iberoamericana [en línea], núm. 1, Moscú, Academia de Ciencias de Rusia, Instituto de Latinoamérica, 1998, $<$ http://www.plugcom.ru/ ilaran/revista $>$, consultada el 10. de marzo de 2000.

- Hettne, Björn y Edmé Dominguez (1996), "In the European Footsteps: NAFTA as a Case of Regionalism", en Weine Karlsson y Axhil Malaki (editores), Growth, Trade and Integration in Latin America, Estocolmo, Institute of Latin American Studies, pp. 149-179.

- Informe Latinoamericano, 5 de agosto de 1997.

- Johnson, Harry (1965), "An Economic Theory of Protectionism, Tariff Bargaining and the Formation of Customs Unions", Journal of Political Econo$m y$, núm. 72, pp. 253-283.

- Lauth, Hans Joachin (1994), "NAFTA y su importancia para América Latina: ¿mayor vinculación o factor de separación”, en Manfred Mols et al., Cambio de paradigmas en América Latina, nuevos impulsos, nuevos temores, Caracas, Nueva Sociedad, pp. 165-176.

- Lipsey, Richard (1998), "Growth, Erosion and Restructuring of the Multilateral Trade System", Paper delivered at the Annual Meeting of the American Economic Association, Atlanta, Georgia, diciembre de 1989.

- - (1960), "The Theory of Customs Unions/A General Survey", The Economic Journal, vol. LXx, núm. 270, febrero, pp. 40-46.

- Lustig, Nora (1994), “NAFTA: Potential Impact on Mexico's Economy and Beyond", en Roberto Bouzas y Jaime Ros (editores), Economic Integration in the Western Hemisphere, Notre Dame, Notre Dame University Press, pp. 46-57.

- Marchal, André (1970), Integración y regionalización de la economía europea, Madrid, Seminarios y Ediciones, S.A. 
- Moneta, Carlos J. (1997), "Espacios económicos e inserción externa: nuevos parámetros, Capítulos SELA, núm. 50, abril-junio, pp. 115-123.

- Ostry, Silvia (1992), "The NAFTA: its International Background", en Stephen Randall et al., North America without Borders, Integrating Canada, The United States and Mexico, Calgary, University of Calgary Press, pp. 21-29.

- Rosas, María Cristina (1994), "Crisis del multilateralismo clásico: política comercial externa de Estados Unidos y zonas de libre comercio", Revista Mexicana de Ciencias Políticas y Sociales, vol. xxxix, núm. 157, nueva época, julio-septiembre, pp. 39-59.

- Salgado, Germánico (1994), "El Grupo Andino: entre dos concepciones de la integración económica”, Síntesis, núm. 24, Madrid, julio-diciembre, pp. 69-92.

- _ (1979), "El Mercado regional latinoamericano: el proyecto y la realidad”, Revista de la CEPAL, Santiago de Chile, abril, pp. 87-133.

- Vacchino, Juan Mario (1982), "Teorías, esquemas y experiencias de integración económica regional”, Mundo Nuevo, año 5, núm. 15-16, Caracas, enero-junio, pp. 154-182.

- Valladão, Alfredo G. A. (1994), Le Retour du Panaméricanisme. La Stratégie des Etats Unis en Amérique Latine après la guerre froide, París, CREST.

- Viner, Jacob (1950), The Customs Union Issue, Nueva York, Carnigie Endwment for Peace.

- Wiarda, Howard (1997), "Consensus Found, Consensus Lost: Disjunctures in US Policy Toward Latin America in the Turn of the Century", Journal of Interamerican Studies and World Affairs, vol. 39, núm. 1, primavera.

- Wonnacott, Ronald (1990), "US Hub and Spoke Bilateral and Multilateral Trading System", CD Howe Commentary, núm. 23, Toronto, octubre.

- __ (1992), "Canadá ante las negociaciones sobre el Libre Comercio entre México y Estados Unidos”, en Gustavo Vega Cánovas (coordinador), México ante el libre comercio con América del Norte, México, El Colegio de México/ Universidad Tecnológica de México, pp. 353-363. 